05

\title{
Пиннинг доменных стенок в двухслойной ферромагнитной нанопроволоке полями рассеяния наночастиц
}

\author{
() О.Л. Ермолаева, В.Л. Миронов \\ Институт фоизики микроструктур РАН, \\ Нижний Новгород, Россия \\ E-mail: ermolaeva@ipmras.ru
}

\begin{abstract}
Приводятся результаты микромагнитного моделирования процессов пиннинга и депиннинга доменной стенки (ДС) в прямоугольной ферромагнитной нанопроволоке (НП), состоящей из двух магнитных слоев, полями рассеяния двух прямоугольных двухслойных наночастиц (НЧ), расположенных по разные стороны от НП и ориентированных перпендикулярно к ее оси. Исследованы особенности перемагничивания данной системы во внешнем магнитном поле в зависимости от направления магнитных моментов слоев наночастиц. Показано, что величина поля депиннинга в такой системе существенно зависит от взаимной ориентации магнитных моментов НЧ и намагниченности НП. Обсуждается возможность реализации магнитной логической ячейки, выполняющей операцию „конъюнкция“ троичной логики.
\end{abstract}

Работа поддержана проектом РФФИ (15-02-04462) и программами президиума РАН.

DOI: 10.21883/FTT.2017.11.45055.14k

\section{1. Введение}

В последнее время проводятся интенсивные исследования управляемого пиннинга ДС в ферромагнитных НП, обусловленные перспективами разработки различных устройств магнитной логики [1-3]. Логические „0“ и „1“ кодируются в таких устройствах как направление намагниченности определенных участков НП, а логические вычисления связаны с процессами движения и пиннинга/депиннига ДС во внешних магнитных полях. Наиболее простым является пиннинг ДС на искусственных ловушках в виде углублений или выступов различной формы на краях и верхней поверхности НП [7-13]. Однако данные ловушки не позволяют осуществлять управление процессами пиннинга/депиннинга и непригодны для создания логических ячеек. Более перспективным является пиннинг ДС с использованием локальных магнитных полей, создаваемых ферромагнитными НЧ, расположенными вблизи НП [14-19]. В этом случае НЧ играют роль магнитного затвора, управляемого внешними полями, что позволяет реализовать на их основе различные варианты логических ячеек. В предыдущих работах нами были предложены различные варианты пиннинга ДС в планарных однослойных ферромагнитных НП-НЧ системах [16-19]. Были рассмотрены особенности движения ДС вдоль НП, связанные с прохождением неоднородного потенциального рельефа, создаваемого полями рассеяния НЧ. Было продемонстрировано, что величина энергии пиннинга ДС определяется взаимной ориентацией магнитных моментов в системе и может существенно изменяться за счет изменения конфигурации магнитных моментов в подсистеме НЧ. Кроме того, обсуждались возможности реализации на основе рассмотренных систем различных вариантов магнитных логических ячеек двузначной логики. Настоящая работа посвящена исследованию особенностей движения и пин- нинга ДС в многослойной системе НП-НЧ. Движение доменных стенок в такой системе, в отличие от ранее рассмотренных однослойных структур, определяется не только взаимодействием НП с полями рассеяния наночастиц, но и магнитостатическим взаимодействием между двумя магнитными слоями самой НП. Кроме того, рассматриваемая система имеет более широкий набор возможных метастабильных состояний, что позволяет, в качестве практического приложения, рассмотреть возможность реализации на ее основе логического элемента троичной логики.

\section{2. Методика расчетов}

Нами была рассмотрена планарная ферромагнитная система, представляющая собой НП и две прямоугольные НЧ, расположенные перпендикулярно оси НП (рис. 1). Структура состоит из двух слоев магнитного материала, разделенных немагнитной прослойкой. На

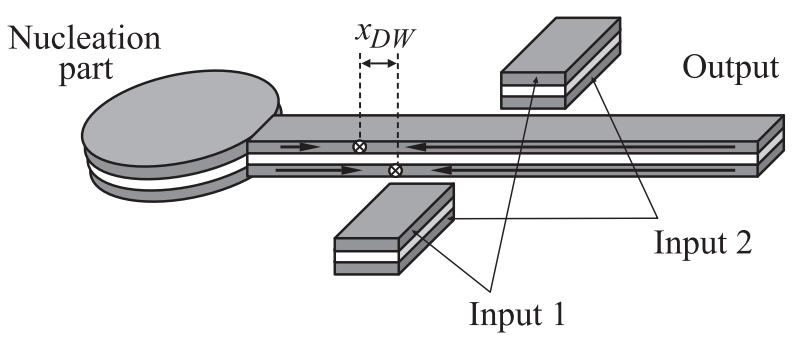

Рис. 1. Многослойная система НП-НЧ. Магнитные слои показаны темным цветом, немагнитная прослойка - белым. Стрелками показана одна из возможных конфигураций магнитной логической ячейки на основе данной системы. $x_{D W}-$ расстояние между доменными стенками в верхнем и нижнем слое. 
одном из концов НП расположено утолщение цилиндрической формы, служащее для контролируемого зарождения ДС [16-19]. При этом в зависимости от соотношения толщины и ширины в НП могут формироваться либо поперечные, либо вихревые ДС [20-22]. В тонких и узких НП легче реализуются поперечные ДС, в более толстых и широких - вихревые.

Особенности процессов перемагничивания системы НП-НЧ во внешнем магнитном поле при различных магнитных конфигурациях намагниченности частиц были изучены при помощи микромагнитного моделирования с использованием программного симулятора Object Oriented MicroMagnetic Framework (OOMMF) [23]. Иcследуемая система имела следующие параметры: латеральные размеры НП $250 \times 3000 \mathrm{~nm}$; размеры частиц $250 \times 500 \mathrm{~nm}$; расстояние между НЧ и НП $50 \mathrm{~nm}$. Диаметр затравочной части $500 \mathrm{~nm}$. Толщина немагнитной прослойки $100 \mathrm{~nm}$. Рассматривались две системы, отличающиеся толщинами магнитных слоев. В системе с толщиной слоев $20 \mathrm{~nm}$ реализовалась поперечная ДС, а в системе с толщиной слоев $40 \mathrm{~nm}$ - вихревая. В качестве магнитного материала рассматривался пермаллой со следующими параметрами: намагниченность насыщения $M_{s}=800 \mathrm{G}$, константа обменного взаимодействия $J=13 \cdot 10^{-7} \mathrm{erg} / \mathrm{cm}$, константа анизотропии $K=0$. Зарождение и движение ДС в НП происходило под действием внешнего реверсивного магнитного поля, приложенного вдоль НП.

\section{3. Результаты}

На первом этапе нами были выполнены оценки энергии взаимодействия ДС, расположенных в соседних магнитных слоях НП без учета полей рассеяния НЧ. При отсутствии обменного взаимодействия между слоями энергия НП с ДС состоит только из магнитостатической энергии взаимодействия между магнитными слоями и зависит от расстояния между ДС $\left(x_{D W}\right)$. Данная энергия может быть записана в следующем виде

$$
E\left(x_{D W}\right)=\int_{V} \int_{V^{\prime}} \mathbf{H}\left(\mathbf{r}-\mathbf{r}^{\prime}\right) \mathbf{M}(\mathbf{r}) d \mathbf{r} d \mathbf{r}^{\prime},
$$

где $\mathbf{H}\left(\mathbf{r}-\mathbf{r}^{\prime}\right)$ - магнитное поле, создаваемое одним слоем НП в области второго, $\mathbf{M}(\mathbf{r})$ - намагниченность второго слоя. Интегрирование проводится по обоим слоям НП. На рис. 2 приведены зависимости энергии системы, состоящей из бесконечной двухслойной НП, для различных типов ДС.

Из рис. 2 видно, что ДС отталкиваются друг от друга. Это соответствует эффекту вытеснения ДС в различных направлениях и формирования в НП состояния с антиферромагнитным упорядочением слоев, соответствующего минимуму энергии. Для противонаправленных поперечных ДС существует локальный минимум энергии при их расположении одна над другой. Он возникает за счет магнитостатического взаимодействия участков

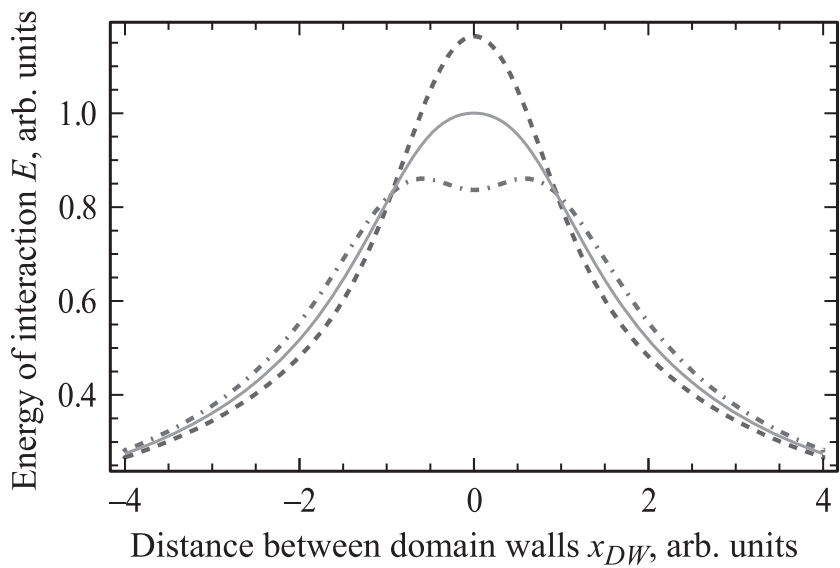

Рис. 2. Нормированные зависимости энергии взаимодействия ДС, находящихся в различных слоях НП, от латерального расстояния между ними. Сплошная линия соответствует вихревым ДС; штриховая линия - сонаправленным, а штрихпунктирная - противонаправленным поперечным ДС в нижнем и верхнем слоях. Энергии взаимодействия нормированы на энергию НП с вихревыми ДС, расположенными одна над другой. По горизонтальной оси отложено расстояние между ДС, нормированное на толщину немагнитной прослойки.

намагниченности, расположенных непосредственно в области ДС верхнего и нижнего слоя.

Движение ДС в системе НП-НЧ под действием магнитного поля имеет ряд особенностей. В качестве примера, на рис. 3 представлены результаты моделирования прохождения под действием магнитного поля поперечной ДС через НЧ-затвор при ориентации магнитных моментов НЧ навстречу друг другу.

Вначале НП однородно намагничивается в направлении справа налево. После снятия поля в слоях затравочной части реализуются вихревые состояния с различным направлением завихренности. Затем к системе прикладывается поле, направленное в противоположную сторону. Поперечные ДС зарождаются одновременно в затравочной части НП в состоянии с противонаправленными моментами. Под действием поля они одновременно движутся к затвору (рис. 3,a), однако в силу отталкивания одна из стенок обгоняет другую (в нашем случае это ДС верхнего слоя). Пиннинг ДС происходит в потенциальной яме [16] в области между НЧ (рис. 3,b). Положение обеих ДС устойчиво вплоть до напряженности магнитного поля $H_{D 1}=210$ Ое. При превышении поля депиннинга $H_{D 1}$ одна из ДС (в рассматриваемом случае верхнего слоя) покидает потенциальную яму и начинает двигаться к свободному концу НП. При этом происходит стабилизация положения второй ДС, в силу того что намагниченности в соседних слоях справа от затвора оказываются в состоянии с антиферромагнитным упорядочением, понижающим энергию системы (рис. 3,c). При достижении напряженности поля $H_{D 2}=250$ Ое происходит депиннинг второй ДС и перемагничивание нижнего слоя (рис. $3, d$ ). 


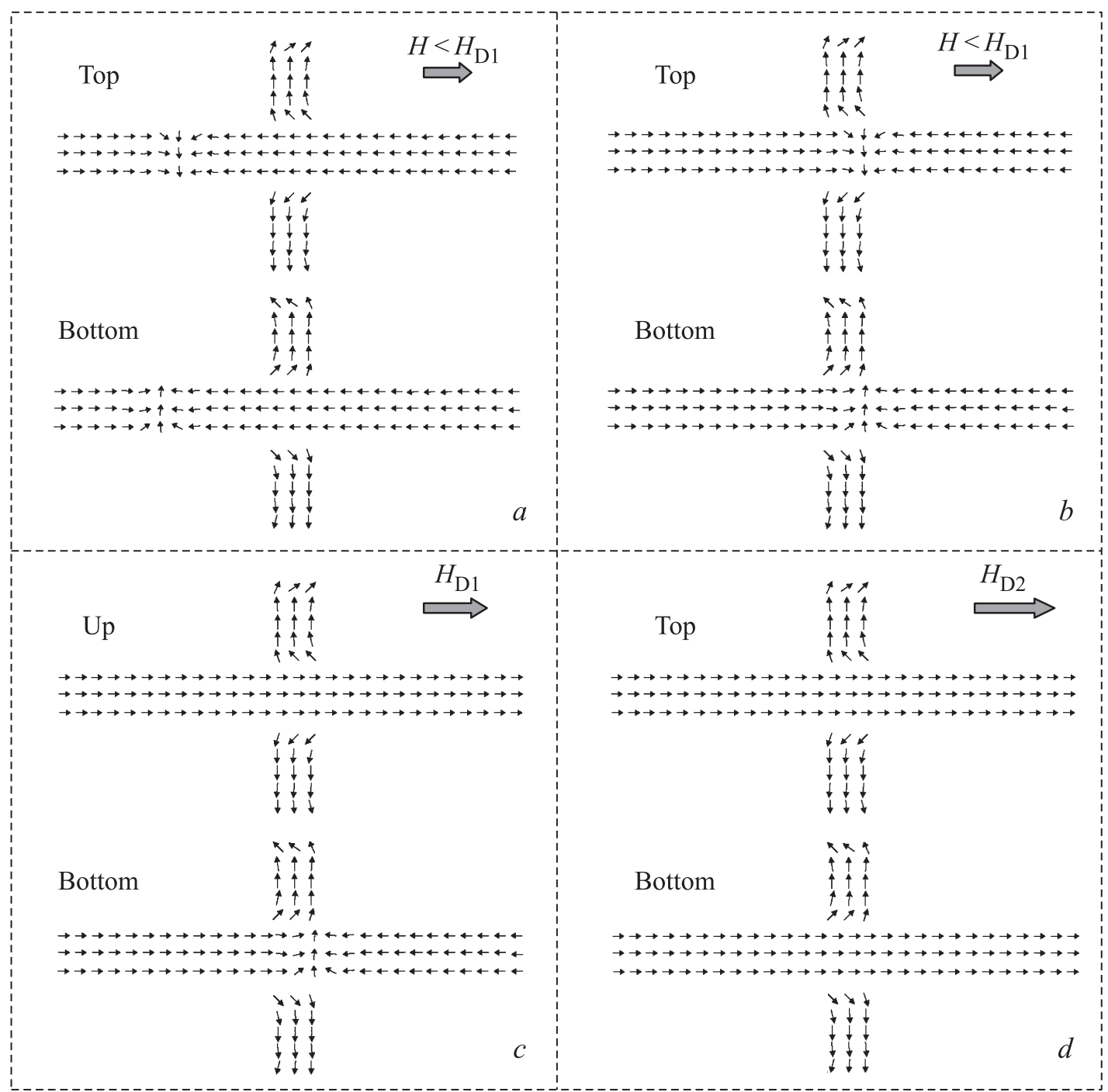

Рис. 3. Последовательные стадии процесса перемагничивания НП толщиной $20 \mathrm{~nm}$ (поперечная ДС) во внешнем магнитном поле.

Процесс перемагничивание системы с вихревой ДС происходит немного сложнее (рис. 4). При приближении ДС к затвору ее структура трансформируется из вихревой в поперечную (рис. $4, b$ ) и пиннинг происходит так же как в рассмотренном выше случае (рис. 4, $c, d)$. При превышении поля депиннинга HD1 ДС вновь трансформируется из поперечной в вихревую (рис. $4, c, e$ ).

Поля рассеяния НЧ создают сложный профиль потенциальной энергии для движения ДС вдоль НП. В Двухслойной системе существует шесть неэквивалентных конфигураций намагниченности НЧ, отличающихся направлением магнитного момента каждого слоя. Данным конфигурациям соответствуют различные профили потенциальной энергии, отличающиеся величиной энергии пиннинга и, соответственно, значениями полей депиннинга ДС.

Для системы НП-НЧ с толщиной магнитных слоев $20 \mathrm{~nm}$ (поперечная ДС) было проведено микромагнитное моделирование процессов перемагничивания для всех неэквивалентных магнитных состояний. На рис. 5 приведены зависимости $x$-компоненты намагниченности, нормированной на величину намагниченности насыщения $M_{x} / M_{s}$ от внешнего магнитного поля $\mathrm{H}$, направленного вдоль оси НП для всех состояний. Стрелками на рисунке схематично показаны направления магнитных моментов в системе НЧ. Из рис. 5 видно, что перемагничивание системы происходит в два этапа при любых конфигурациях намагниченности НЧ.

Для рассмотренных параметров системы величины полей депиннинга соотносятся следующим образом:

$$
\begin{gathered}
H_{4}<H_{21}<H_{22}<H_{31}<H_{11}<H_{12}<H_{31}<H_{32} \\
<H_{61}<H_{41}<H_{52}<H_{62} .
\end{gathered}
$$

\section{4. Обсуждение}

На основе рассматриваемой системы может быть реализована магнитная логическая ячейка, выполняющая 


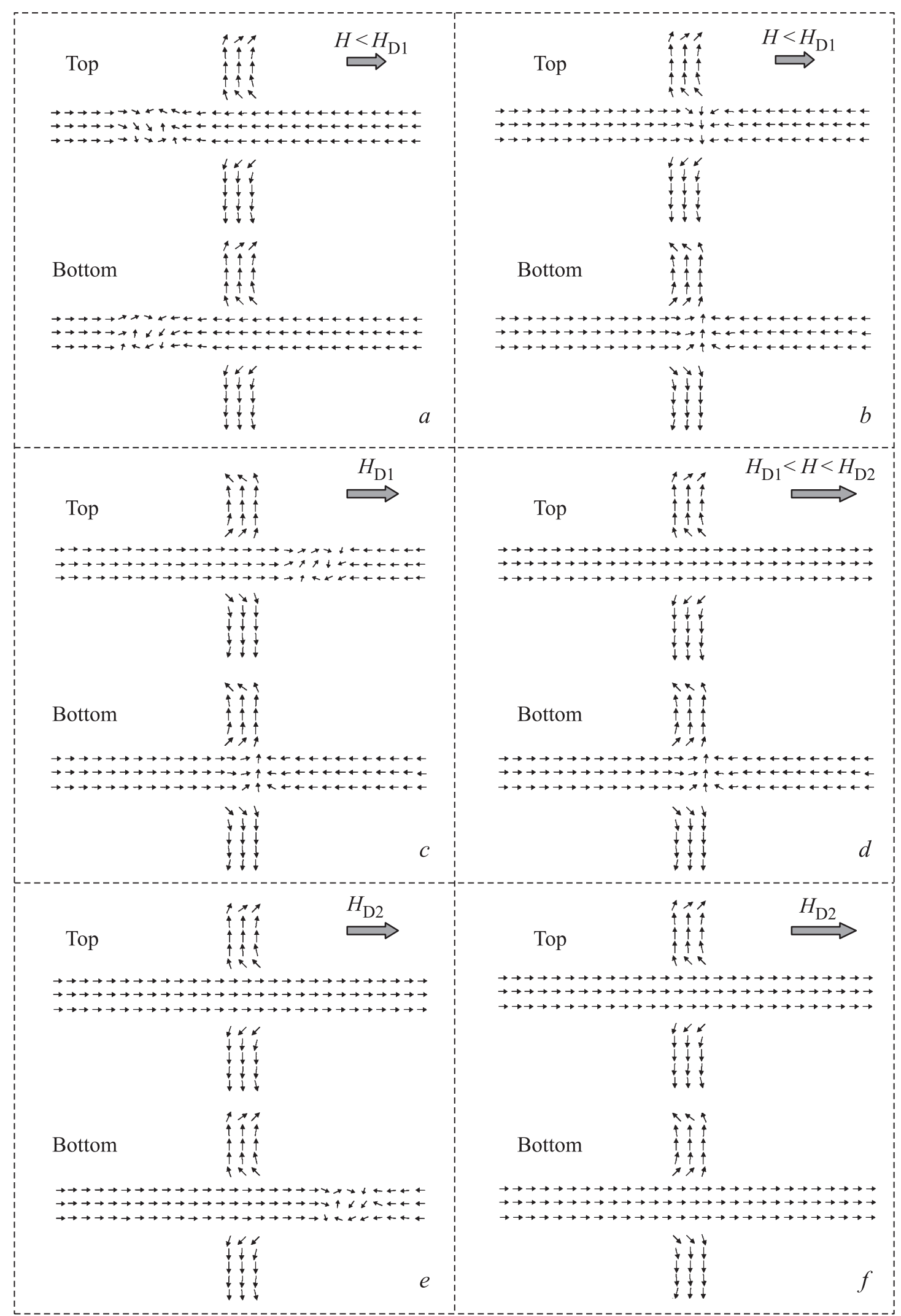

Рис. 4. Последовательные стадии процесса перемагничивания нанопроволоки с вихревой доменной стенкой во внешнем магнитном поле. 

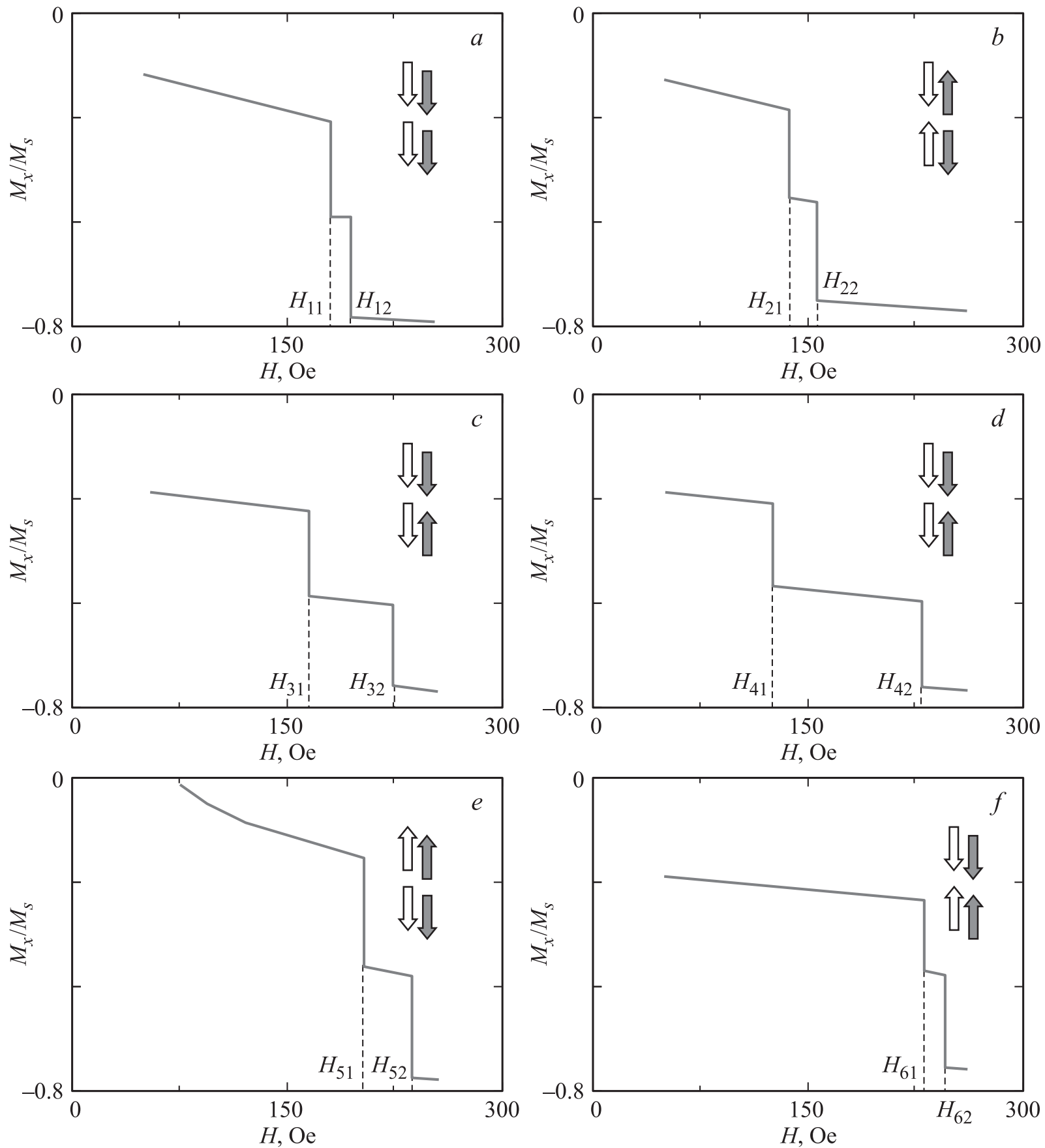

Рис. 5. Кривые перемагничивания НП для различных конфигураций намагниченности НЧ. Светлыми стрелками показана намагниченность в верхнем слое НЧ, темными стрелками — в нижнем слое.

операции троичной логики. Троичная логика, в отличие от двоичной, определяет операции над множеством значений „истинно“, „неопределенно“ и ,ложно“, которое также может быть записано в виде „1“, „0“ и ,-1“.

Обозначим верхние слои двух НЧ как „Вход 1“, а нижние слои как „Вход 2“. Кроме того, обозначим свободный конец НП как „Выход“ (рис. 1). Тогда можно поставить в соответствие направлениям намагниченности в слоях различных элементов логические переменные в соответствии с табл. 1 .
Таблица 1. Соответствие между конфигурациями магнитных моментов НЧ (Вход), а также свободного конца НП (Выход), и логическими переменными

\begin{tabular}{l|c|c|c}
\hline $\begin{array}{l}\text { Логические } \\
\text { переменные }\end{array}$ & -1 & 0 & 1 \\
\hline Вход & $\downarrow$ & $\uparrow$ & $\uparrow$ \\
Выход & $\uparrow$ & $\uparrow$ & $\downarrow$ \\
\hline
\end{tabular}


Таблица 2. Соответствие значений входов и выхода для операции „коньюнкция“ троичной логики

\begin{tabular}{l|r|r|r|r}
\hline \multicolumn{2}{c|}{$\begin{array}{c}\text { Логические } \\
\text { переменные }\end{array}$} & -1 & 0 & 1 \\
\cline { 2 - 5 } \multicolumn{2}{c|}{ Вход 1 } \\
\hline \multirow{2}{*}{ Вход 2 } & -1 & 1 & 0 & -1 \\
& 0 & 0 & 0 & 0 \\
& 1 & -1 & 0 & 1
\end{tabular}

При такой конфигурации входов на основе данной системы реализуется ячейка, выполняющая операцию „коньюнкция“ (логическое умножение) троичной логики, у которой соответствие между логическими значениями входов и выхода устанавливается в соответствии с табл. 2.

Работа логической ячейки состоит из нескольких этапов. На первом этапе оба слоя НП намагничиваются однородно (справа налево), так что магнитное состояние соответствует логической „1“ на выходе ячейки. На втором этапе вводится входная информация посредством намагничивания НЧ. На третьем этапе к ячейке прикладывается инвертированное тестирующее магнитное поле $H_{T}$, которое вызывает зарождение ДС и ее движение к магнитному затвору. В зависимости от направления магнитных моментов частиц ДС либо пиннингуется в области затвора, либо проходит через затвор в одном или в обоих слоях. Для корректной работы ячейки величина тестирующего магнитного поля должна лежать в пределах $H_{11}<H_{T}<H_{12}$. На последнем этапе происходит считывание выходной информации. Затем цикл вычислений повторяется.

\section{5. Заключение}

Таким образом, в настоящей работе были изучены особенности пиннинга ДС в ферромагнитной многослойной планарной системе, представляющей собой НП и две расположенные перпендикулярно ей НЧ. Было показано, что величина поля депиннинга в такой системе существенно зависит от взаимной ориентации магнитных моментов НЧ и намагниченности НП. Проанализированы неэквивалентные магнитные конфигурации системы, отличающиеся величиной энергии пиннинга ДС и соответственно значениями поля депиннинга. Полученные результаты указывают на возможность создания на основе рассмотренной системы магнитной логической ячейки, выполняющей операцию „коньюнкция“ троичной логики.

\section{Список литературы}

[1] L. O’Brien, D.E. Read, H.T. Zeng, E.R. Lewis, D. Petit, R.P. Cowburn. Appl. Phys. Lett. 95, 232502 (2009).

[2] H.T. Zeng, D.E. Read, L. O’Brien, J. Sampaio, E.R. Lewis, D. Petit, R.P. Cowburn. Appl. Phys. Lett. 96, 262510 (2010).
[3] R. Mattheis, S. Glathe, M. Diegel, U. Hubner. J. Appl. Phys. 111, 113920 (2012).

[4] H.T. Zeng, D. Petit, L. O’Brien, D. Read, E.R. Lewis, R.P. Cowburn. J. Magn. Magn. Mater. 322, 2010 (2010).

[5] L. O’Brien, D. Petit, E.R. Lewis, R.P. Cowburn, D.E. Read, J. Sampaio, H.T. Zeng, A.-V. Jausovec. Phys. Rev. Lett. 106, 087204 (2011).

[6] M. Hayashi, L. Thomas, R. Moriya, C. Rettner, S.S.P. Parkin. Science 320, 209 (2008).

[7] E.R. Lewis, D. Petit, L. Thevenard, A.V. Jausovec, L. O'Brien, D.E. Read, R.P. Cowburn. Appl. Phys. Lett. 95, 152505 (2009).

[8] D. Petit, A.V. Jausovec, D.E. Read, R.P. Cowburn. J. Appl. Phys. 103, 114307 (2008).

[9] D. Petit, A.V. Jausovec, H.T. Zeng, E.R. Lewis, L. O’Brien, D.E. Read, R.P. Cowburn. Phys. Rev. B 79, 214405 (2009).

[10] K. O'Shea, S. McVitie, J.N. Chapman, J.M.R. Weaver. Appl. Phys. Lett. 93, 202505 (2008).

[11] L.K. Bogart, D. Atkinson, K. O'Shea, D. McGrouther, S. McVitie. Phys. Rev. B 79, 054414 (2009).

[12] E. Varga, G. Csaba, A. Imre, W. Porod. IEEE Trans. Nanotechnol. 11, 682 (2012).

[13] Q. Zhu, X. Liu, S. Zhang, Q. Zheng, J. Wang, Q. Liu. Jpn. J. Appl. Phys. 53, 073001 (2014).

[14] S.M. Ahn, K.W. Moon, C.G. Cho, S.B. Choe. Nanotechnology 22, 085201 (2011).

[15] R.A. van Mourik, C.T. Rettner, B. Koopmans, S.S.P. Parkin. J. Appl. Phys. 115, 17D503 (2014).

[16] V.L. Mironov, O.L. Ermolaeva, E.V. Skorohodov, A.Yu. Klimov. Phys. Rev. B 85, 144418 (2012).

[17] В.Л. Миронов, О.Л. Ермолаева. Изв. РАН. Сер. физ. 78, 36 (2014).

[18] V.L. Mironov, O.L. Ermolaeva, E.V. Skorohodov. IEEE Trans. Magn. 52, 1100607 (2016).

[19] О.Л. Ермолаева, Е.В. Скороходов, В.Л. Миронов. ФТТ 58, 2145 (2016).

[20] R.D. McMichael, M.J. Donahue. IEEE Trans. Magn. 33, 4167 (1997).

[21] Y. Nakatani, A. Thiaville, J. Miltat. J. Magn. Magn. Mater. 290-291, 750 (2005).

[22] M. Klaui, C.A.F. Vaz, J.A.C. Bland, L.J. Heyderman, F. Nolting, A. Pavlovska, E. Bauer, S. Cherifi, S. Heun, A. Locatelli. Appl. Phys. Lett. 85, 5637, (2004).

[23] M.J. Donahue, D.G. Porter. OOMMF User's Guide. Interagency Report NISTIR 6376. National Institute of Standards and Technology, Gaithersburg, MD (1999). http://math.nist.gov/oommf. 